\title{
Hematite at its thinnest limit
}

\author{
C. Bacaksiz, M. Yagmurcukardes, F. M. Peeters, and M. V. Miloševił* \\ Theory of Functional Materials, Department of Physics, \\ University of Antwerp, Groenenborgerlaan 171, B-2020 Antwerp, Belgium and \\ NANOlab Center of Excellence, University of Antwerp, Belgium
}

\begin{abstract}
Motivated by the recent synthesis of two-dimensional $\alpha-\mathrm{Fe}_{2} \mathrm{O}_{3}$ [Balan et al. Nat. Nanotech. 13, $602(2018)]$, we analyze the structural, vibrational, electronic and magnetic properties of singleand few-layer $\alpha-\mathrm{Fe}_{2} \mathrm{O}_{3}$ compared to bulk, by ab-initio and Monte-Carlo simulations. We reveal how monolayer $\alpha-\mathrm{Fe}_{2} \mathrm{O}_{3}$ (hematene) can be distinguished from the few-layer structures, and how they all differ from bulk through observable Raman spectra. The optical spectra exhibit gradual shift of the prominent peak to higher energy, as well as additional features at lower energy when $\alpha-\mathrm{Fe}_{2} \mathrm{O}_{3}$ is thinned down to a monolayer. Both optical and electronic properties have strong spin asymmetry, meaning that lower-energy optical and electronic activities are allowed for the single-spin state. Finally, our considerations of magnetic properties reveal that 2D hematite has anti-ferromagnetic ground state for all thicknesses, but the critical temperature for Morin transition increases with decreasing sample thickness. On all accounts, the link to available experimental data is made, and further measurements are prompted.
\end{abstract}

\section{INTRODUCTION}

Following the successful isolation of graphene, $\frac{112}{, 2}$ an immense effort has been exerted on the synthesis of other two dimensional (2D) layered materials such as group III$\mathrm{V}$ binary compounds $(h \text {-BN, } h \text {-AlN })^{3 / 7}$ and transitionmetal dichalcogenides (TMDs) $\stackrel{[-15}{[15}$ Most of these 2D materials have bulk counterparts that are formed by van der Waals (vdW) stacked layers. Beside those, the atomically thin layers of silicene $e^{16 / 17}$ and germanene, ${ }^{[16}$ which have non-layered bulk forms, have been successfully synthesized. Over the last years, the synthesis of ultra-thin materials from their non-layered bulk counterparts has also attracted interest in the material science, $\frac{18 \sqrt{20}}{\text { where }}$ one of the most recent successes is the extraction of ultrathin $\alpha-\mathrm{Fe}_{2} \mathrm{O}_{3}$ (2D hematite) $\underline{21}$

Iron oxide is a well-studied material family including several structural phases. The family exhibits various electronic and magnetic properties, such as band gap ranging from insulator to semiconductor, $\frac{222}{26}$ superparamagnetism, 27 and weak ferromagnetism.28129131 34 Iron oxide has four different crystalline phases at ambient pressures: $\alpha-\mathrm{Fe}_{2} \mathrm{O}_{3}$ (hematite), $\beta-\mathrm{Fe}_{2} \mathrm{O}_{3}, \gamma-\mathrm{Fe}_{2} \mathrm{O}_{3}$ (maghemite), and $\varepsilon-\mathrm{Fe}_{2} \mathrm{O}_{3}$. Among these phases, hematite is a thermodynamically stable polymorph of $\mathrm{Fe}_{2} \mathrm{O}_{3}$, and a well-known ferromagnet that undergoes magnetic phase transitions from paramagnetic to weakferromagnetic state at the Neel temperature $\left(T_{N}\right)$ of 961 $\mathrm{K}$, and from weak-ferromagnetic to anti-ferromagnetic state at Morin transition temperature $\left(T_{M}\right)$ of $265 \mathrm{~K} \cdot 28 \mid 29$ In addition, it has optimal band gap for light absorption applications, $\frac{30}{30}$ and has been widely investigated for several other technological applications such as gas sensing, 25135 lithium-ion batteries, $\frac{36-38}{38}$ water treatment, $, 49,41$ and catalysis. 42

It is already well established that nano-structuring of a material can significantly influence its magnetic and other properties due to quantum confinement and surface effects. ${ }^{43}$ Such effects on $\alpha$ - $\mathrm{Fe}_{2} \mathrm{O}_{3}$ were also stud- ied. Schroeer et al. reported that $T_{M}$ of microcrystal hematite is depressed under negative pressure induced due to the lattice spacing. ${ }^{45}$ Zysler et al. demonstrated that $T_{M}$ increases as the size of hematite nanoparticles increases ${ }^{44}$ In addition, Sorescu et al. studied the weak ferromagnetic phase above $T_{M}$ and an anti-ferromagnetic phase below $T_{M}$ for different sizes and morphologies of hematite nanoparticles. ${ }^{46}$ Only recently, the focus of research shifted to the effects of thickness, after Balan et al. synthesized atomically-thin hematite and reported that it exhibits optical band gap comparable with the bulk sample, and weak ferromagnetism at low temperature regime without any Morin transition.21 In their report, two different 2D hematite samples were considered, exfoliated from [001] and [010] crystallographic directions, and [001] 2D samples were claimed to be more stable based on the molecular dynamics simulations. They also attempted to support the experimental results by a DFT study, however, they considered only on a monolayer structure, which was not present in experiment at all. In the meantime, $2 \mathrm{D} \alpha-\mathrm{Fe}_{2} \mathrm{O}_{3}$ (named hematene) was reported in experimental and in DFT-based study as a stable anti-ferromagnetic semiconductor ${ }^{47 / 48}$ same as bulk, which further clouds the conclusions regarding thickness dependence of the magnetic properties of hematite in its thinnest limit.

Motivated by above issues, and recognizing the importance of hematene as a newest member of the family of magnetic 2D materials (rapidly emerging after the synthesis of the first magnetic single-layer of $\mathrm{CrI}_{3}{ }^{49}$ that may have many other uses as well, we here thoroughly investigate its thickness-dependent physical properties, namely structural, vibrational, electronic, and magnetic properties of monolayer, few-layer, and bulk $\alpha-\mathrm{Fe}_{2} \mathrm{O}_{3}$ by performing density functional theory-based calculations and Monte-Carlo simulations. In doing so, we reveal how the atomistic thickness of $\alpha-\mathrm{Fe}_{2} \mathrm{O}_{3}$ is clearly reflected in the Raman and visible range optical spectra, and the critical temperature, all relevant to the ongoing experi- 
mental efforts in the field.

The paper is organized as follows. Details of the computational methodology are presented in Sec. III. The thickness-dependent structural, vibrational, electronic, optical, and magnetic properties of hematene versus fewlayer and bulk hematite are then comparatively discussed in Secs. III AIIE We summarize our results and conclusions in Sec. IV]

\section{COMPUTATIONAL METHODOLOGY}

To investigate the structural, vibrational, magnetic, and electronic, properties of a two-dimensional $\alpha-\mathrm{Fe}_{2} \mathrm{O}_{3}$ crystal, first-principle calculations were performed in the framework of density functional theory (DFT) as implemented in the Vienna ab-initio simulation package (VASP) $!^{[5151}$ The Perdew-Burke-Ernzerhof (PBE) ${ }^{52}$ form of the generalized gradient approximation (GGA) was adopted to describe electron exchange and correlation. The Hubbard $U$ term was included to be $4 \mathrm{eV}$ for Fe atom in all calculations to account for the strong onsite Coulomb interaction. ${ }^{54}$ The van der Waals (vdW) correction to the GGA functional was included by using the DFT-D2 method of Grimme. 53 The charge transfer in the system was determined by the Bader technique $[55$

The kinetic energy cut-off for plane-wave expansion was set to $600 \mathrm{eV}$ and the energy was minimized until its variation in the following steps became lower than $10^{-6}$ $\mathrm{eV}$. The Gaussian smearing method was employed for the total energy calculations. In order to capture the correct occupancy of the surface states, the width of smearing was set to $0.01 \mathrm{eV}$ (checked to be sufficiently small for few-layer $\mathrm{Fe}_{2} \mathrm{O}_{3}$ samples). Total Hellmann-Feynman forces was taken to be $10^{-5} \mathrm{eV} / \AA$ for the structural optimization. $24 \times 24 \times 1 \Gamma$-centered $k$-point sampling was used in the primitive unit cells. To avoid interaction between successive layers in the out-of-plane direction, we used a vacuum spacing of $12 \AA$ A. Regarding the dielectric function calculations, $48 \times 48 \times 1 \Gamma$-centered $k$-point sampling was used and direct $(q=0)$ single-particle excitations from valence to conduction band were calculated. The local field effects were included at DFT level.

In order to investigate the dynamical stability of a monolayer hematene structure, the phonon band dispersions were calculated by the small displacement method, as implemented in the PHON code ${ }^{56}$ In addition, the first order off-resonant Raman activities of the phonon modes at the $\Gamma$ point were obtained by calculating the change in the macroscopic dielectric tensor with respect to the normal mode describing each vibrational mode using finite-difference method $[57$ A detailed theory of the Raman scattering can be found in our recent studies 58159

To investigate the magnetic exchange parameters between the magnetic sites, we used four-state methodology ${ }^{60}$ which relies on mapping of the energetics of different magnetic configurations onto Heisenberg spin
Hamiltonian:

$$
H=\sum_{\langle i j\rangle_{n}}-J_{n}^{i j} S_{i} \cdot S_{j}
$$

where $J_{n}^{i j}$ is the magnetic exchange parameter between $i$ th and $j$ th magnetic sites, indexed by $n$ in the nearestneighbor sequence. Considering two atomic sites, 1 and 2 , which are the nearest neighbors of each other and the corresponding exchange parameter, $J_{1}^{12}$, the energy can be written as:

$$
E=J_{1}^{12} S_{1} \cdot S_{2}+S_{1} \cdot \mathrm{K}_{1}+S_{2} \cdot \mathrm{K}_{2}+E_{\text {others }} .
$$

Here the first term describes the interaction between sites 1 and $2, \mathrm{~K}_{1}=\sum_{\langle 1 j \neq 1,2\rangle_{n}} J_{n}^{1 j} S_{1}$ and $\mathrm{K}_{2}=$ $\sum_{\langle 2 j \neq 1,2\rangle_{n}} J_{n}^{2 j} S_{2}$ are the interactions of site 1 with others and site 2 with others, respectively. Last term stands for the interaction between the sites different from 1 and 2. To isolate $J_{1}^{12}$, four different magnetic configurations were chosen as (i) $S_{1}=S, S_{2}=S$; (ii) $S_{1}=-S, S_{2}=S$; (iii) $S_{1}=S, S_{2}=-S$; (iv) $S_{1}=-S, S_{2}=-S$, and $S_{\text {other }}=S$, so that

$$
\begin{aligned}
& E^{(\mathrm{i})}=E_{\text {others }}+J_{1}^{12} S^{2}+S^{2} K_{1}+S^{2} K_{2}, \\
& E^{(\mathrm{ii})}=E_{\text {others }}-J_{1}^{12} S^{2}-S^{2} K_{1}+S^{2} K_{2}, \\
& E^{(\mathrm{iii})}=E_{\text {others }}-J_{1}^{12} S^{2}+S^{2} K_{1}-S^{2} K_{2}, \\
& E^{(\mathrm{iv})}=E_{\text {others }}+J_{1}^{12} S^{2}-S^{2} K_{1}-S^{2} K_{2} .
\end{aligned}
$$

This set of equations yields $J_{1}^{12}$ as

$$
J_{1}^{12}=\frac{E^{(\mathrm{i})}+E^{(\mathrm{iv})}-E^{(\mathrm{ii})}-E^{(\mathrm{iii})}}{4 S^{2}},
$$

which is a general formula for any considered pair of magnetic sites.

The temperature-dependent magnetization of the system was then estimated by performing standard Metropolis Monte-Carlo (MC) simulations on top of the DFT-calculated magnetic exchange parameters, $J_{n}$ 's. Regarding the MC simulations, $4 \times 4 \times 1$ lattice with periodic boundary conditions in the lateral directions (outof-plane periodicity was considered for bulk) was constructed. Randomly generated spin configuration was cooled down from 2500 to $0 \mathrm{~K}$, where at each temperature $2 \times 10^{3}$ spin-flips per site were executed to obtain thermal equilibrium.

\section{RESULTS AND DISCUSSION}

\section{A. Thickness-dependent structural properties}

The well-known bulk $\alpha-\mathrm{Fe}_{2} \mathrm{O}_{3}$ structure belongs to $R \overline{3} 2 / c$ space group in which ABC stacked buckled hexagonal sublayers of $\mathrm{Fe}$ atoms have octahedral coordination with six $\mathrm{O}$ atoms as shown in Fig. 1(a). In-plane and outof-plane lattice parameters are found to be $a=b=5.08$ 

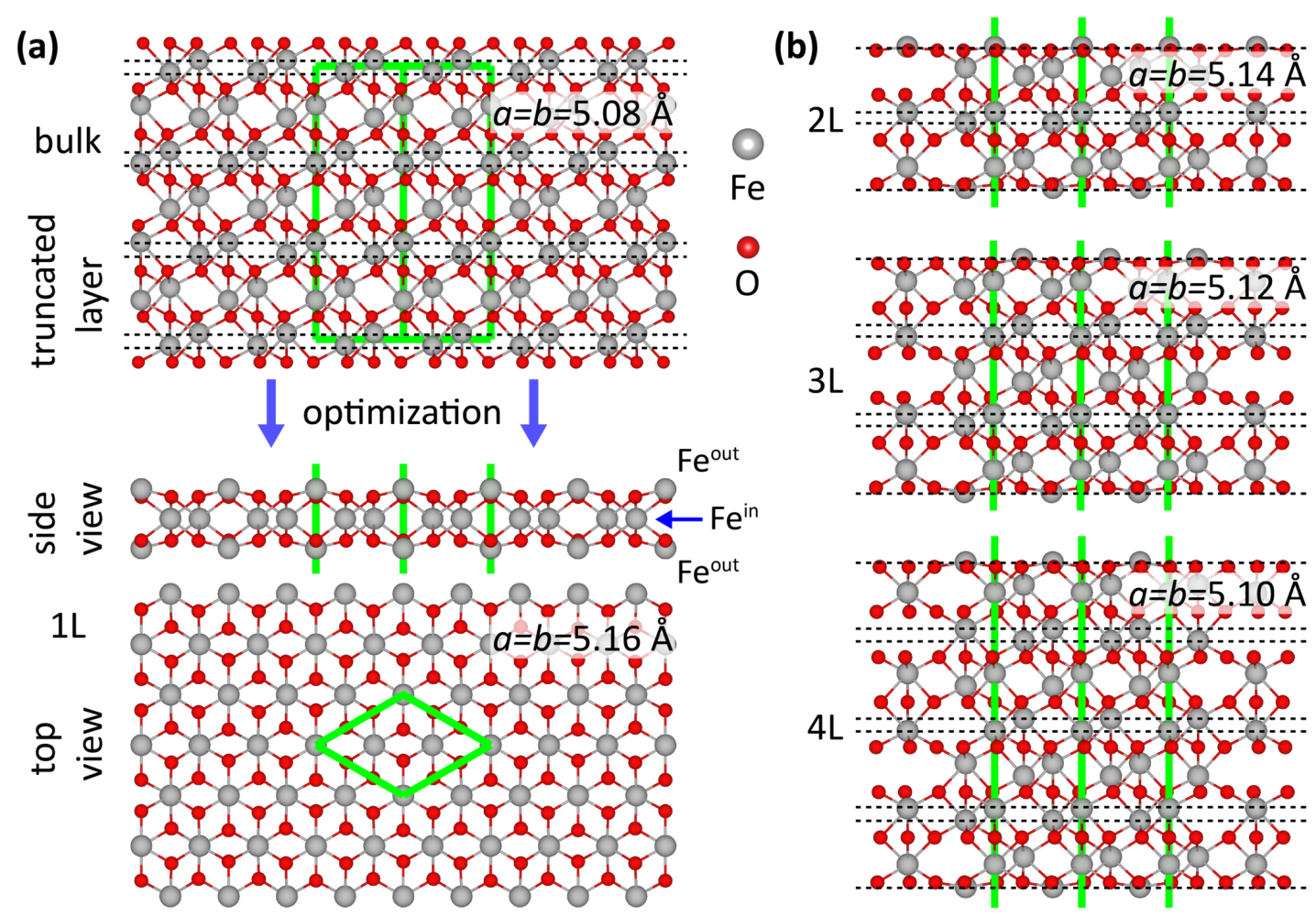

FIG. 1: (color online) (a) The side and top views of hematene truncated from the bulk $\alpha$-Fe $\mathrm{O}_{3}$ in the [001] direction, after optimization. Green line denotes the primitive unit cell. Two types of Fe atoms are also indicated as $\mathrm{Fe}^{\text {in }}$ and Fe ${ }^{\text {out }}$. (b) The side view of the optimized structure of $2 \mathrm{~L}, 3 \mathrm{~L}$, and $4 \mathrm{~L} \alpha-\mathrm{Fe}_{2} \mathrm{O}_{3}$.

Åand $c=13.72 \AA$, as listed in Table I. Since Balan et al. reported that atomically thin layers exfoliated in [001] directions are more stable ${ }^{21}$ we consider the thicknessdependent properties for [001] hematite samples. As an initial structure, we truncated a layer from the bulk in the [001] direction, as shown by dashed lines in Fig. 1(a). The unit cell of the truncated layer consists of four Fe and six $\mathrm{O}$ atoms which fits the formula unit of $\mathrm{Fe}_{2} \mathrm{O}_{3}$. Differing from the bulk form, there are two types of Fe atoms due to the absence of the coexisting atoms in the out-

TABLE I: In-plane lattice constants, $a$ and $b$; out-of-plane lattice constant for bulk, $c$; the average distance between $\mathrm{Fe}^{\text {out }}$ atoms per number of layers, $d$; the electron donation per $\mathrm{Fe}$ atom, $\Delta \rho$, the first and second value discriminating $\mathrm{Fe}^{\mathrm{in}}$ and $\mathrm{Fe}^{\text {out }}$, respectively; the magnetic ground state, MS; the cohesive energy per unit formula, $E_{\mathrm{c}}$; the energy band gap (GGA with $U=4 \mathrm{eV}), E_{\text {gap }}$.

\begin{tabular}{|c|c|c|c|c|c|c|c|c|}
\hline & $\begin{array}{c}a \\
(\AA)\end{array}$ & $\begin{array}{c}b \\
(\AA)\end{array}$ & $\begin{array}{c}c \\
(\AA)\end{array}$ & $\begin{array}{c}d \\
(\AA)\end{array}$ & $\begin{array}{c}\Delta \rho \\
\left(e^{-}\right)\end{array}$ & $\begin{array}{c}\text { MS } \\
-\end{array}$ & $\begin{array}{c}E_{\mathrm{c}} \\
(\mathrm{eV})\end{array}$ & $\begin{array}{l}E_{\text {gap }} \\
(\mathrm{eV})\end{array}$ \\
\hline Bulk & 5.08 & 5.08 & 13.72 & - & 1.8 & AFM & 5.00 & 2.10 \\
\hline $4 \mathrm{~L}$ & 5.10 & 5.10 & - & 4.12 & $1.8 / 1.6$ & AFM & 4.90 & M \\
\hline $3 \mathrm{~L}$ & 5.12 & 5.12 & - & 3.95 & $1.8 / 1.6$ & AFM & 4.87 & M \\
\hline $2 \mathrm{~L}$ & 5.14 & 5.14 & - & 3.60 & $1.8 / 1.6$ & AFM & 4.81 & M \\
\hline $1 \mathrm{~L}$ & 5.16 & 5.16 & - & 3.04 & $1.8 / 1.6$ & AFM & 4.62 & 0.75 \\
\hline
\end{tabular}

of-plane direction: inner Fe atoms with the octahedral bond coordination, labeled $\mathrm{Fe}^{\mathrm{in}}$, and the outer Fe atoms with the trigonal pyramidal coordination, labeled $\mathrm{Fe}^{\text {out }}$ (see Fig. 11(a)).

The optimized 1L structure (hematene), shown in Fig. 1(a), has a perfectly symmetric hexagonal sublattice of $\mathrm{Fe}^{\mathrm{in}}$ atoms sandwiched by directly stacked two trigonal sublattices of $\mathrm{Fe}^{\text {out }}$ atoms. The octahedral coordination of $\mathrm{Fe}^{\text {in }}$ and trigonal pyramidal coordination of $\mathrm{Fe}^{\text {out }}$ atoms remain such. The space group of the new $2 \mathrm{D}$ structure is $P \overline{3} 12 / m$. As given in Table $\mathrm{I}$, the lattice parameters are found to be $a=b=5.16 \mathrm{~A}$, slightly larger than those of bulk. The distance between the $\mathrm{Fe}^{\text {out }}$ atoms is $d=3.04 \AA$, smaller than $3.95 \AA$ in bulk. It is also found that each $\mathrm{Fe}^{\text {in }}$ atom donates $1.8 e^{-}$to an $\mathrm{O}$ atom. $\mathrm{Fe}^{\text {out }}$ donates $1.6 e^{-}$which is slightly smaller due to having coordination 3 instead of 6 .

Differing from the monolayer, the structural configurations and the bond coordination of the thicker systems, $2 \mathrm{~L}, 3 \mathrm{~L}$, and $4 \mathrm{~L}$, remain similar to those of bulk $\alpha-\mathrm{Fe}_{2} \mathrm{O}_{3}$, as shown in Fig. 1(b). Slight structural changes presented in Table I indicate that adjacent layer(s) in the out-of-plane direction prevent atoms from rearranging their position as obtained in the optimization of the $1 \mathrm{~L}$ structure. 
(a)

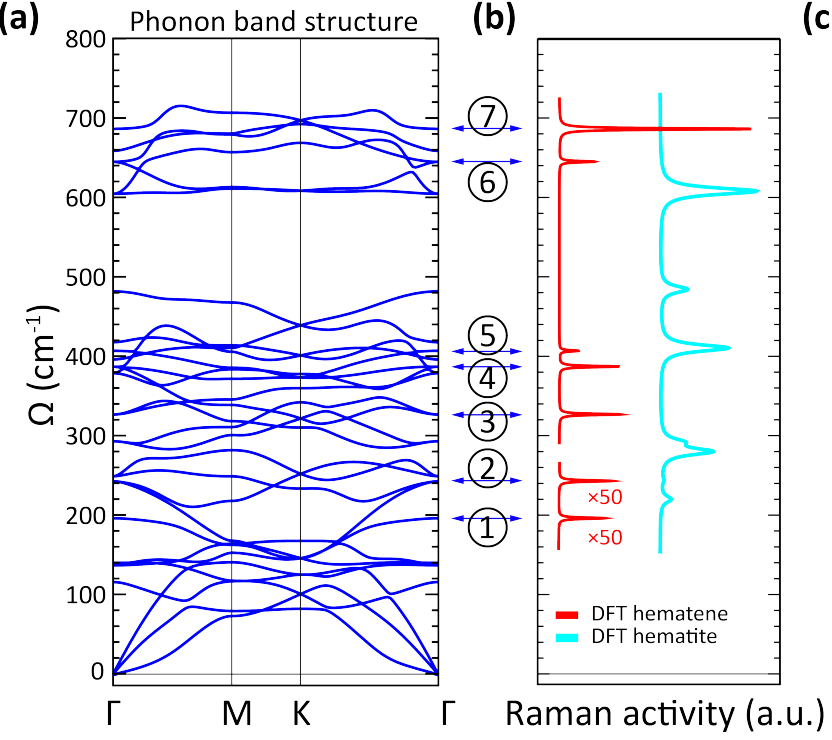

(c)
(7)

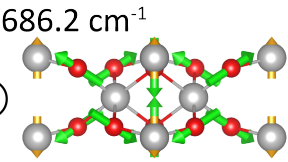

(6)

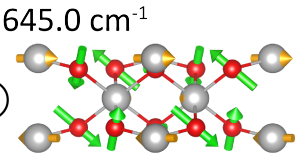

(5)

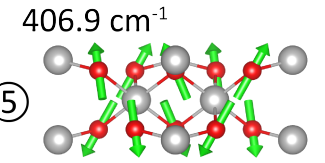

(4)

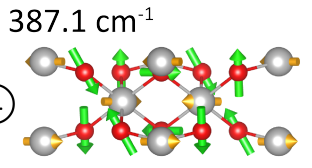

(3)

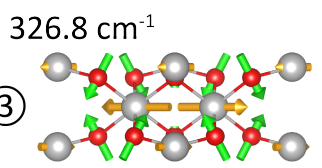

$243.0 \mathrm{~cm}^{-1}$

(2)

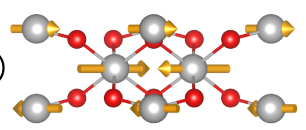

(1)

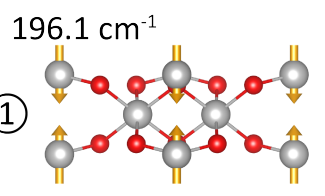

FIG. 2: (color online) (a) Phonon band structure of hematene through high symmetry points of the BZ. (b) Calculated Raman spectrum for hematene and bulk hematite (red and turquoise, respectively). (c) The vibrational characteristics of Raman active phonon modes of hematene.

\section{B. Thickness-dependent vibrational properties}

The dynamical stability of the optimized $1 \mathrm{~L} \alpha-\mathrm{Fe}_{2} \mathrm{O}_{3}$ is examined by calculating its phonon band dispersion through the high symmetry points of the Brillouin zone (BZ), as shown in Fig. 2(a). It is evident that hematene is dynamically stable, and exhibits thirty phonon branches - three of which are acoustic. The highest frequency of the optical phonon branches at the $\Gamma$ point is found to be $686.2 \mathrm{~cm}^{-1}$, which is relatively large as compared to those of $\mathrm{MX}_{2}$ where $\mathrm{M}=\mathrm{Mo}, \mathrm{W}$, Re and $\mathrm{X}=\mathrm{S}$, Se (401.0-450.0 $\left.\mathrm{cm}^{-1}\right) ! 58$ Having optical phonon branches at higher frequency indicates the relatively high mechanical stiffness of $1 \mathrm{~L} \alpha-\mathrm{Fe}_{2} \mathrm{O}_{3}$. For illustrative comparison, we also give the values of graphene $\left(1555.0 \mathrm{~cm}^{-1}\right)$ and $h$-BN $\left(1343.4 \mathrm{~cm}^{-1}\right)$.

For further elucidation of the vibrational properties, the first order off-resonant Raman activities of the phonon modes for $1 \mathrm{~L} \alpha-\mathrm{Fe}_{2} \mathrm{O}_{3}$ are calculated and presented in Fig. 2(b). There are seven Raman active phonon modes which are assigned as 1-to-7 from low to high frequencies (see Fig. 2(c)). The seven Raman active modes can be categorized as three non-degenerate out-of-plane (having frequencies 196.1, 406.9, and 686.2 $\mathrm{cm}^{-1}$ ) and four doubly-degenerate in-plane vibrational modes (with frequencies of 243.0, 326.8, 387.1, and 645.0 $\mathrm{cm}^{-1}$ ). The mode at frequency $196.1 \mathrm{~cm}^{-1}$ has the lowest Raman activity and is attributed to the purely outof-plane breathing-like vibration of Fe atoms (see Fig. 2 (c)). Another non-degenerate phonon mode at the frequency of $406.9 \mathrm{~cm}^{-1}$ demonstrates the mostly out-ofplane vibration of $\mathrm{O}$ atoms while $\mathrm{Fe}$ atoms make no contribution. The highest frequency optical mode is found at $686.2 \mathrm{~cm}^{-1}$, which is the most prominent peak and represents the purely out-of-plane vibration of Fe atoms and mostly in-plane vibration of $\mathrm{O}$ atoms. Notably, this phonon mode can only be observed in $2 \mathrm{D} \alpha-\mathrm{Fe}_{2} \mathrm{O}_{3}$ structures and is absent in bulk (or unobservable due to its negligibly small Raman activity). The doubly-degenerate phonon mode at $243.0 \mathrm{~cm}^{-1}$ has a low Raman activity and it is attributed to the in-plane vibration of Fe atoms against each other, while $\mathrm{O}$ atoms do not contribute to the vibration. The mode at $326.8 \mathrm{~cm}^{-1}$ arises from the in-plane opposite vibration of $\mathrm{Fe}$ atoms while $\mathrm{O}$ atoms vibrate in opposite out-of-plane directions at the top and the bottom of the layer. A similar vibrational character is found for the phonon mode at frequency $387.1 \mathrm{~cm}^{-1}$, in which adjacent $\mathrm{O}$ atoms vibrate in opposite out-of-plane directions. Finally, the phonon mode at $645.0 \mathrm{~cm}^{-1}$ has similar vibrational character with the mode at frequency $387.1 \mathrm{~cm}^{-1}$. In this mode, the $\mathrm{Fe}^{\mathrm{in}}$ atoms have relatively small contribution to the vibration.

The calculated Raman spectrum of bulk $\alpha-\mathrm{Fe}_{2} \mathrm{O}_{3}$ reveals also seven Raman active peaks. Two out-of-plane non-degenerate phonon modes are assigned as $\mathrm{A}_{1 g}$ modes at the frequencies of 220.0 and $484.3 \mathrm{~cm}^{-1}$. Other five Raman active modes having frequencies 244.0, 280.0, $292.1,410.4$, and $607.9 \mathrm{~cm}^{-1}$ are doubly-degenerate and are assigned as $\mathrm{E}_{g}$ modes.

In Fig. 3, we present our results on the Raman spectra of $\alpha-\mathrm{Fe}_{2} \mathrm{O}_{3}$ structures from monolayer to bulk, with comparison to the experimental observations reported by Balan et al..${ }^{211}$ Since the intensities of the peaks are relatively low at the frequencies below $350 \mathrm{~cm}^{-1}$, we zoom in on the spectra in $150-350 \mathrm{~cm}^{-1}$ range in the left panel of the figure, where peak-intensities are multiplied by given factors to facilitate the observation of the frequency-shift. The dashed lines track the peak-positions with respect to 
the experimental peak-positions on the frequency axis. In general, the calculated Raman spectrum of the bulk shows good agreement with the experiment of Ref 21 . For the 2-4L samples, the calculated Raman spectra capture the main features of the experimental spectrum of a quasi-2D structure, with slightly stronger shift of the peak frequencies as compared to the experimental shift upon exfoliation. However, monolayer $\mathrm{Fe}_{2} \mathrm{O}_{3}$ exhibits distinctive peak positions. Balan et al. demonstrated in their thickness measurement the absence of samples as thin as a monolayer of either [001] (3.04 $\AA)$ or [010] (3.09 $\AA$ ) phases; our results for Raman response of a monolayer corroborate that fact, as experimentally measured Raman spectrum is completely different from the Raman spectrum expected for a monolayer.

We start the discussion with the peaks in the range $150-350 \mathrm{~cm}^{-1}$, zoomed out in the left panel of Fig. 3 . The frequency of the $\mathrm{A}_{g}$ mode displays phonon hardening with increasing thickness. Its frequency in a monolayer $\left(196.1 \mathrm{~cm}^{-1}\right)$ hardens to $211.5 \mathrm{~cm}^{-1}$ in $2 \mathrm{~L} \alpha-\mathrm{Fe}_{2} \mathrm{O}_{3}$, and further to 212.7 and $214.3 \mathrm{~cm}^{-1}$ in $3 \mathrm{~L}$ and $4 \mathrm{~L}$ structures, respectively. Since this mode is attributed to the vibration of $\mathrm{Fe}^{\text {out }}$ atoms, the change in its frequency decreases with increasing thickness. Therefore, for sufficiently thick samples its frequency is very close to the bulk case. Notably, this behavior is rather different from a layered $(\mathrm{vdW})$ material, because in a non-layered material, the

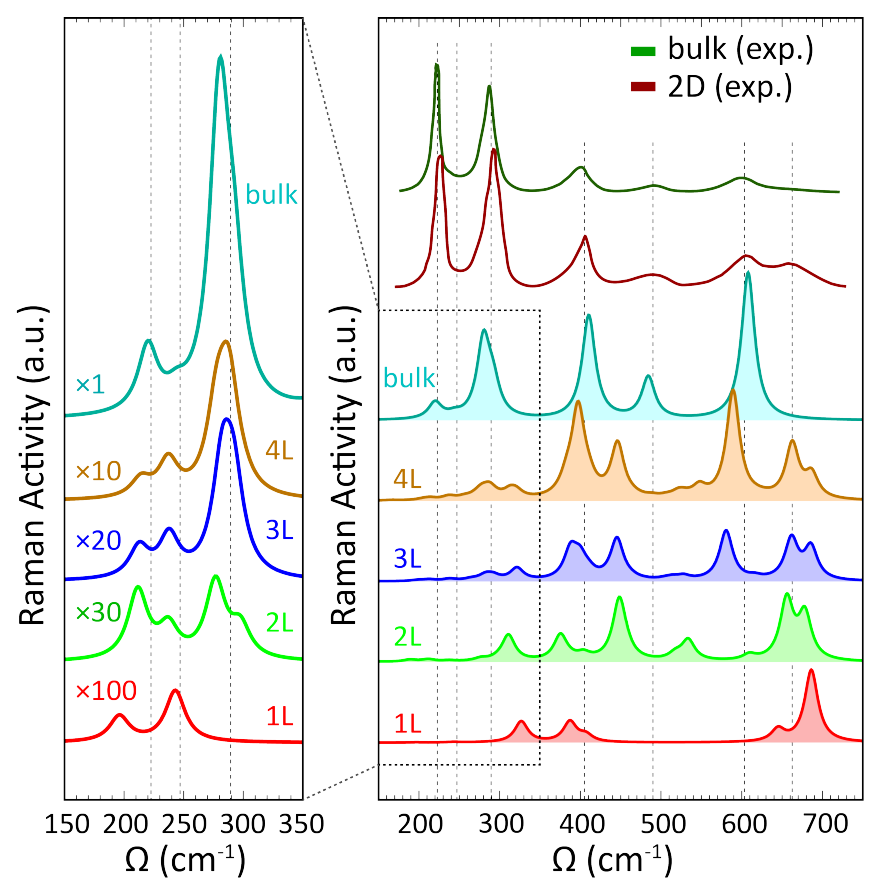

FIG. 3: (color line) Calculated Raman spectrum for 1L, 2L, 3L, 4L, and bulk. Above them, the experimental Raman spectrum for bulk and $2 \mathrm{D} \alpha-\mathrm{Fe}_{2} \mathrm{O}_{3}$ is shown, as reported in Ref 21. The left panel zooms in on the low-intensity peaks in the frequency range $150-350 \mathrm{~cm}^{-1}$. The intensities are multiplied by a given factor to enhance the visibility of the peaks. coordination, length, and strength of the bonds, also the surface reconstructions, may change significantly with thickness. As compared to the experiment, 21 our finding indicates that a very small frequency shift of $\mathrm{A}_{g}$ mode compared to bulk is a signature of a few-layer thick sample. Contrarily, the bulk out-of-plane mode at frequency $484.3 \mathrm{~cm}^{-1}$ displays phonon softening as the structure is thinned down to 2L. Its frequency is found to be 457.1, 463.5 , and $465.4 \mathrm{~cm}^{-1}$ in $2 \mathrm{~L}, 3 \mathrm{~L}$, and $4 \mathrm{~L}$ structures, respectively. In the case of $\mathrm{E}_{g}$ modes, phonon hardening is mostly found with increasing thickness. The mode at frequency $276.7 \mathrm{~cm}^{-1}$ (in 2L structure) displays phonon hardening to $292.1 \mathrm{~cm}^{-1}$ (in bulk) and it is attributed to the in-plane shear vibration of the Fe atoms. Moreover, the phonon mode having frequency $533.6 \mathrm{~cm}^{-1}$ in $2 \mathrm{~L}$ crystal hardens to $607.9 \mathrm{~cm}^{-1}$ in bulk crystal. Notably, those two $\mathrm{E}_{g}$ modes are entirely absent in the 1L case. On the other hand, two particularly Raman active phonon modes at high frequencies appear in 2D structures, while being absent in the bulk crystal. Those two modes are found to be attributed to the mixed in- and out-of-plane vibrations of $\mathrm{O}$ atoms while the $\mathrm{Fe}$ atoms vibrate in-plane or out-of-plane (modes 6 and 7 in Fig. 2(c)). The phonon mode at $645.0 \mathrm{~cm}^{-1}$ in $1 \mathrm{~L}$ hardens to $655.7,661.6$, and $662.6 \mathrm{~cm}^{-1}$ in $2 \mathrm{~L}, 3 \mathrm{~L}$, and $4 \mathrm{~L}$ structures, respectively. It is clear that the increment of the phonon frequency decreases with layer thickness. However, the Raman activity of the phonon mode decreases as the structure becomes thicker, and disappears for bulk hematite. Notably, these two phonon modes exhibit distinctive features for distinguishing of $2 \mathrm{D}$ structures from bulk, since they predominantly arise from vibrations of surface atoms. It is rather remarkable that the phonon frequency differences between bulk and exfoliated samples reported by Balan et al. closely resemble those in our results down to $2 \mathrm{~L}$ structure ${ }^{62}$ However, we also show that [001] monolayer hematene exhibits a completely distinctive Raman spectrum from the thicker structures, which may serve as a tool to distinguish true monolayer hematene from few-layer hematite in future experiments.

\section{Thickness-dependent electronic properties}

The calculated electronic band structure of $1 \mathrm{~L} \alpha-\mathrm{Fe}_{2} \mathrm{O}_{3}$ is shown in Fig. 4(a). The band structure has asymmetric dispersion depending on the spin component. The blue curve (denoting up-spin, $S_{\uparrow}$ ), has its valence band maximum (VBM), which is also global maximum, at the $K$ point. The $S_{\uparrow}$ component shows mid-gap states, dispersive over the energy axis between $0.75-0.94 \mathrm{eV}$ in all directions of the BZ, indicating that the mid-gap band is conductive with minimum at the $\Gamma$ point. Therefore, the conduction band minimum (CBM) of the $S_{\uparrow}$ component is the global minimum and makes hematene an indirect band-gap semiconductor with a band gap of $0.75 \mathrm{eV}$. In addition, regardless of the mid-gap states, the conduc- 


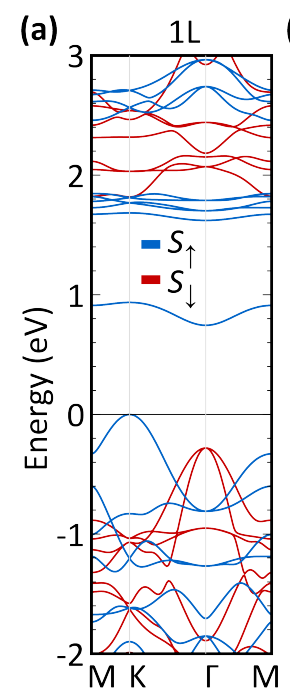

(b) Total
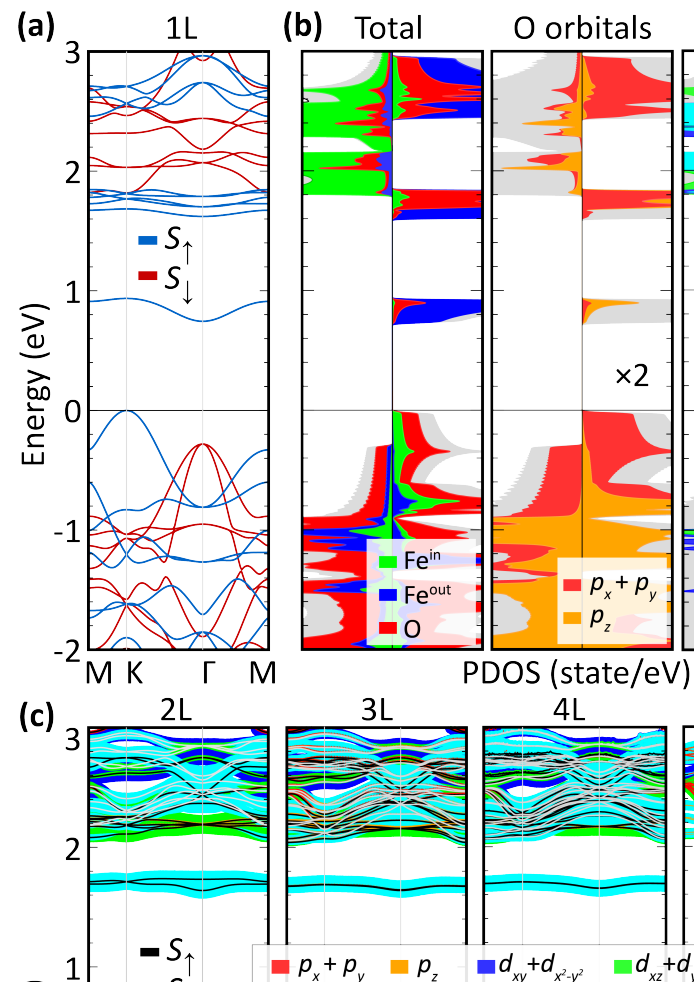

bulk

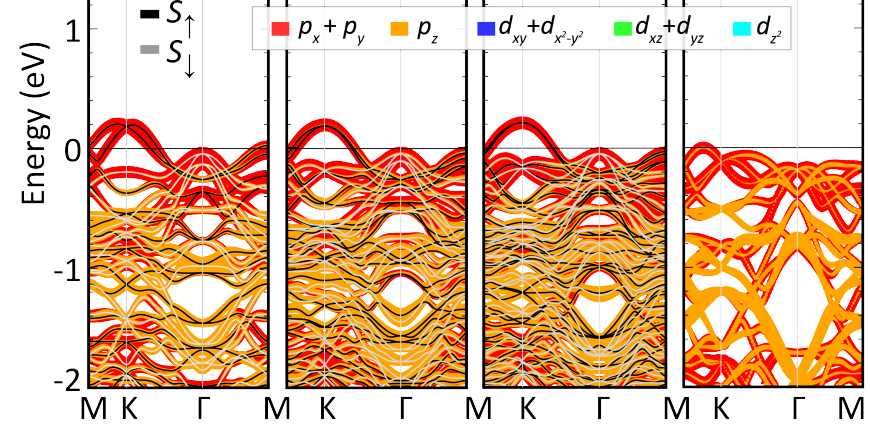

FIG. 4: (color line) (a) The spin-polarized electronic band structure of $1 \mathrm{~L} \alpha-\mathrm{Fe}_{2} \mathrm{O}_{3}$. (b) Atom- (left) and orbitaldecomposed partial density of states (pDOS) of $1 \mathrm{~L} \alpha-\mathrm{Fe}_{2} \mathrm{O}_{3}$ , with color codes given in the graphs. The grey regions are the total density of state given as a reference for comparison. (c) Orbital-decomposed electronic band structure of $2 \mathrm{~L}, 3 \mathrm{~L}$, $4 \mathrm{~L}$, and bulk $\alpha-\mathrm{Fe}_{2} \mathrm{O}_{3}$.

tion band of the $S_{\uparrow}$ component has a minimum also at the $K$ point with energy of $1.62 \mathrm{eV}$. Note that all extreme points including the mid-gap band originate from a single spin component of $S_{\uparrow}$.

On the other hand, the down-spin $\left(S_{\downarrow}\right)$ component, with corresponding red lines in Fig. 4(a), possesses its valence band maximum with two degenerate light and heavy hole bands at the $\Gamma$ point. The VBM of the $S_{\downarrow}$ component appears at the $M$ point with an energy of $-0.28 \mathrm{eV}$ with respect to Fermi-level. The conduction band of the $S_{\downarrow}$ component at the $M$ point is at $1.82 \mathrm{eV}$, giving rise to a band gap of $S_{\downarrow}$ component of $2.10 \mathrm{eV}$.

For further analysis of the electronic properties, the atom- and orbital-decomposed partial density of states (pDOS) of $1 \mathrm{~L} \alpha-\mathrm{Fe}_{2} \mathrm{O}_{3}$ was calculated. In the left panel of Fig. 4(b), the atom-decomposed pDOS for $\mathrm{Fe}^{\mathrm{in}}, \mathrm{Fe}^{\text {out }}$, and $\mathrm{O}$ atoms is shown. The VBM, in which only the $S_{\uparrow}$ states exist, is dominated by the oxygen states and considerable amount of states originate from only $\mathrm{Fe}^{\mathrm{in}}$ atoms. For the $S_{\downarrow}$ part, most of the states are from oxygen, yet only the states of $\mathrm{Fe}^{\text {out }}$ atoms have contributions. The mid-gap band, on the other hand, is dominated by the $\mathrm{Fe}^{\text {out }}$ states and there is a small contribution from the oxygen states.

In central and right panels of Fig. 4(b), the pDOS for $p$-orbitals of $\mathrm{O}$ atoms and $d$-orbitals of Fe atoms are shown, respectively. It is revealed that the $\mathrm{O}$ domination of the VBM (central panel), originates from the $p_{x}$ and $p_{y}$ orbitals, while most of the Fe states (right panel) stem from in- and out-of-plane hybrid orbitals of $d_{x z}$ and $d_{y z}$, with small contributions from $d_{x y}$ and $d_{x^{2}-y^{2}}$ orbitals. The $S_{\downarrow}$ part of the valence band has a similar picture considering the $\mathrm{O}$ orbitals, however, the only contribution of Fe atoms are from $d_{x y}$ and $d_{x^{2}-y^{2}}$ orbitals.

The mid-gap states, on the other hand, originate mostly from the $d_{z^{2}}$ orbitals of $\mathrm{Fe}^{\text {out }}$ atoms, as shown in left and right panels of Fig. 4(b), which is a typical example of the conductive surface states with a single chanel. The higher energies of the $\mathrm{CB}$ consist of the combination of $p$-orbitals of $\mathrm{O}$ atoms and $d$-orbitals o Fe.

In order to describe the change of the electronic properties with thickness, we calculate the orbital-decomposed electronic band structures of $2-4 \mathrm{~L}$ and bulk $\alpha$ - $\mathrm{Fe}_{2} \mathrm{O}_{3}$ shown in Fig. 4(c). It is clearly seen that all fewlayer structures have bands crossing the Fermi level which makes them metallic. These bands are dominated by $p_{x}$ and $p_{y}$ orbitals of $\mathrm{O}$ atoms. Note that in order to obtain accurate occupancies of the states around the Fermi-level in a layer exfoliated from a non-van der Waals material, the smearing parameter should be carefully chosen. If the smearing parameter is inappropriately large, the dangling bonds can be occupied and the Fermi-level can be shifted into the band gap, leading to incorrect conclusions about the electronic properties of the sample []$^{63}$ Therefore, we decreased our smearing parameter down to $0.01 \mathrm{eV}$, which allowed us to capture the dangling bonds of $\mathrm{O}$ atoms in $2-4 \mathrm{~L}$ samples.

Regarding the higher energy features of the band structures, all few-layer systems exhibit mid-gap bands, dominated mainly by $d_{z^{2}}$ orbitals of $\mathrm{Fe}$ atoms, and the spin states are split. The bulk system, on the other hand, has degenerate spin states in the band structure. It is clear that the anisotropic spin channels, the mid-gap states and the states crossing the Fermi level in few-layer structures are a consequence of the surface.

\section{Thickness-dependent optical properties}

In order to investigate the optical properties, we calculated the dielectric function of bulk and 1-4L $\alpha-\mathrm{Fe}_{2} \mathrm{O}_{3}$, by considering the direct excitation between the singleparticle states. Then we compare the results with the 


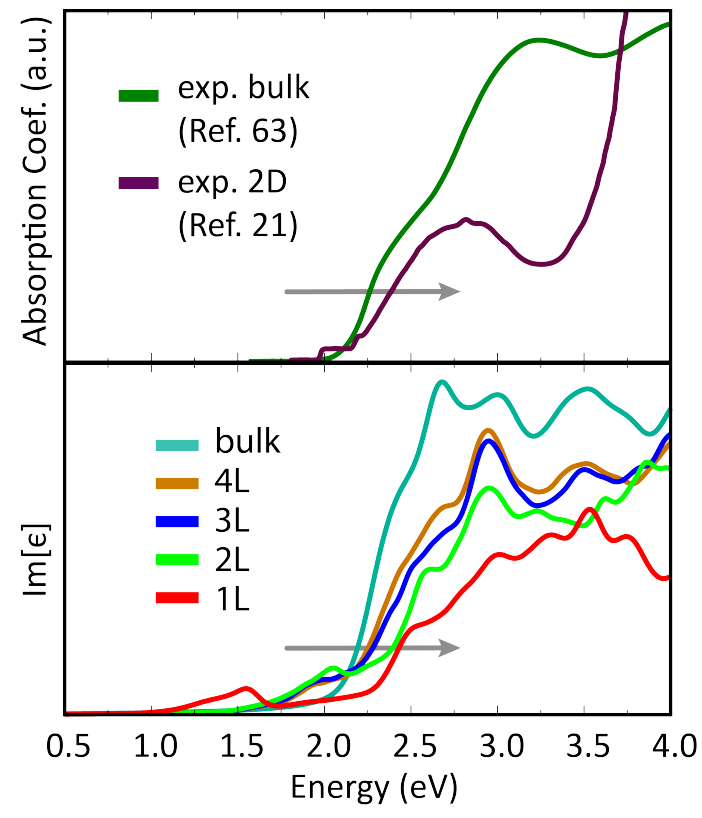

FIG. 5: (color online) Top panel: The experimentallyobtained absorption coefficients for bulk and 2D sample. Bottom panel: Calculated dielectric functions of $1 \mathrm{~L}, 2 \mathrm{~L}, 3 \mathrm{~L}, 4 \mathrm{~L}$, and bulk. Arrow indicates the direction of change with thinning the sample.

experimental measurements on bulk ${ }^{61}$ and $2 \mathrm{D}^{21}$ samples, shown in Fig. 5. Note that the optical gaps taken from the measured and from the calculated optical spectra for a bulk system perfectly match, which yields confidence in the theoretical methodology to directly and reliably compare the calculated spectra of 1-4L systems with the experimentally obtained spectrum of a $2 \mathrm{D}$ sample.21

In general, the experimental spectra of bulk and 2D structure exhibit two main features. (i) When the structure thinned down from bulk to $2 \mathrm{D}$, the prominent features shift to higher energy. That shift is also clearly seen in our calculated spectra. (ii) $2 \mathrm{D}$ spectrum exhibits a small step-like feature at the lower-energy edge of the prominent peak, while the bulk peak rises abruptly which are also confirmed by the calculated spectra.

Looking deeper in the data, the calculated spectrum of hematene (red line) displays its lowest transition at $\sim 1.5$ $\mathrm{eV}$, which is only possible when the mid-gap states of $1 \mathrm{~L}$ shown in Fig. 4 are optically active. From pDOS analysis, we infer that the peak at $\sim 1.5 \mathrm{eV}$ stems mainly from the optical transitions between $p_{x}-p_{y}$ states of oxygen atoms at $\sim-0.4 \mathrm{eV}$, and $d_{z^{2}}$ of $\mathrm{Fe}^{\text {out }}$ at $\sim 0.75-0.94$, which correspond to mid-gap band. Recalling the results of the previous section, it is revealed here that those transitions are possible for the single-spin state. With its distinct mid-gap peak, the calculated spectrum for hematene does not match the experimentally seen absorption spectrum for $2 \mathrm{D} \alpha-\mathrm{Fe}_{2} \mathrm{O}_{3}$ in Ref 21, corroborating the fact that the $2 \mathrm{D}$ structure in experiment was thicker. As shown in our data, the thicker structures of $2-4 \mathrm{~L}$ exhibit step-like fea- ture at $\sim 1.9 \mathrm{eV}$, coinciding with the lower energy edge of the prominent peak. Similarly to mid-gap peak of $1 \mathrm{~L}$, those step-like features originate from the transitions between occupied $p_{x}$ and $p_{y}$ orbitals of oxygen and mid-gap $d_{z^{2}}$ orbital of surface Fe atoms. Note that for 2-4L structures there are bands which are crossing the Fermi-level due to dangling bonds of $\mathrm{O}$ atoms which is shown in Fig. 4(c), however, there is no optical transition found from the occupied bands to those dangling states which are just above the Fermi-level. Therefore, $2-4 \mathrm{~L} \alpha-\mathrm{Fe}_{2} \mathrm{O}_{3}$ has optical band gap, even though it has metallic features originating from optically inactive surface states.

This optical spectrum analysis therefore reveals the clear distinction between the spectra of few-layer and monolayer samples, and also between few-layer and bulk hematite. Our calculations also confirm that experimental realization of $2 \mathrm{D} \alpha-\mathrm{Fe}_{2} \mathrm{O}_{3}$ to date was in $2 \mathrm{~L}$ and thicker forms, and not a monolayer.

\section{E. Thickness-dependent magnetic properties}

In this section, we discuss the thickness-dependent magnetic properties of $1-4 \mathrm{~L}$ and bulk $\alpha-\mathrm{Fe}_{2} \mathrm{O}_{3}$. We first investigate the ground-state magnetic states for all structures by comparing the energetics of the different possible magnetic configurations. Each Fe atom has the magnetic moment of $|m|=4 \mu_{\mathrm{B}}$ in the ground state for all structures. It is found that bulk $\alpha-\mathrm{Fe}_{2} \mathrm{O}_{3}$ has an antiferromagnetic (AFM) ground state shown in Fig. 6(a), which agrees with the earlier results in literature ${ }^{47 / 48}$ Basically, magnetization of $\mathrm{Fe}$ atoms in the hexagonal buckled sublayers are parallel. The sublayers have antiparallel magnetization with respect to each other. The same configuration is also found as the ground state for the $2-4 \mathrm{~L} \alpha-\mathrm{Fe}_{2} \mathrm{O}_{3}$. The state for $1 \mathrm{~L}$ is shown in Fig. 6(a) next to bulk, and is also resembling the state truncated from the bulk AFM state.

The energetics of different magnetic configurations also allows us to calculate the magnetic exchange parameters between the magnetic sites. We map the energetics to Heisenberg spin Hamiltonian given in Eq. (1) by applying the nearest-neighbor (NN) approximation following the procedure given in Sec. II] In the bulk structure, as depicted in Fig. 6(a), the magnetic sites are identical in terms of number of $n$th nearest neighbors and the corresponding bond length. However, for 1-4L structures, the number of $n$th nearest neighbors and the bond lengths differ depending on the position of the magnetic site in question in the out-of-plane direction. Therefore, we performed different sets of calculations for the magnetic exchange parameters experienced by an atom, depending on its position in the sample. The obtained values are listed in Table

In order to obtain the magnetic properties beyond the ground state, we performed MC simulations based on Ising model, using the determined exchange parameters, to predict the temperature-dependent magnetic 
(a) Bulk

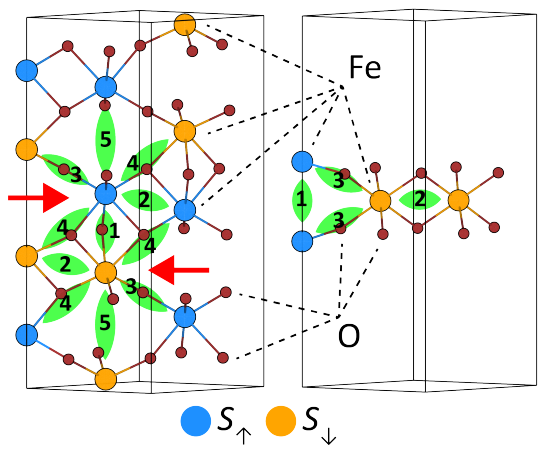

(b)

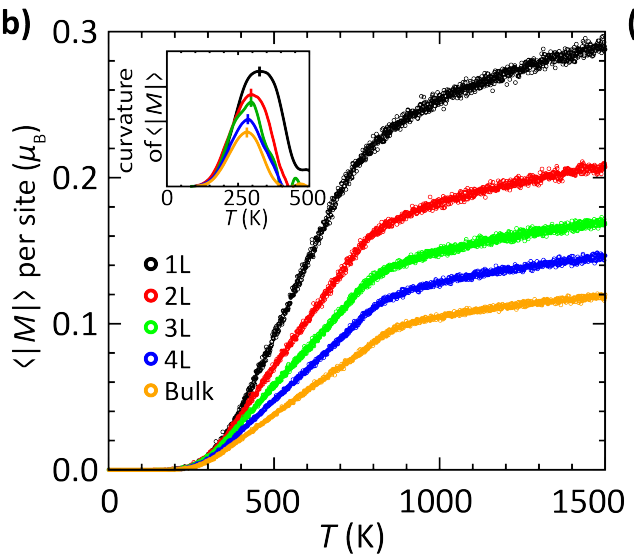

(c)

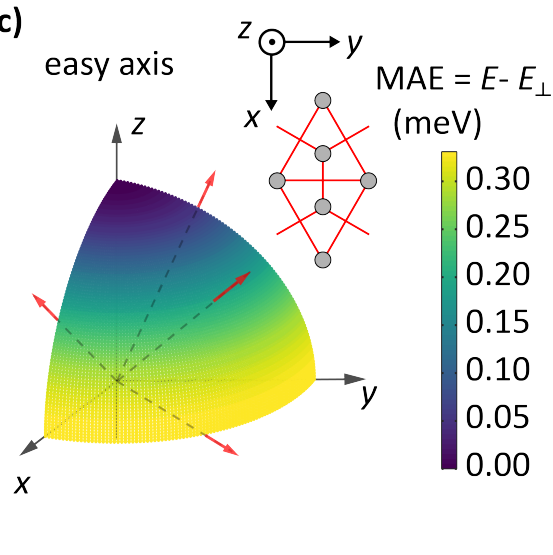

FIG. 6: (color line) (a) Schematic illustration of the magnetic exchange parameters between Fe atoms for bulk (left) and 1L (right). Shown numbers are the indices of the exchange coefficients $J_{n}$ in Eq. (1). (b) The averaged absolute magnetization of 1-4L and bulk systems as a function of temperature. Inset shows $T_{c}$ as a function of thickness, obtained as the temperature of the maximal curvature of the corresponding magnetization curve. (c) The magnetic anisotropy energies of monolayer hematene are projected on a spherical surface, at which normal vectors correspond to magnetization direction, as shown by the arbitrarily placed red arrows.

properties such as critical temperature $\left(T_{c}\right)$. However, the Ising model considers only up/down collinear spins, hence yields much overestimated barriers for magnetic transitions. As a consequence, our trial MC calculation using DFT-obtained exchange parameters for the bulk system yield much larger $T_{c}$ for ferromagnetic to anti-

TABLE II: The magnetic exchange parameters for monolayer, few-layer, and bulk hematite, $J_{n}$, where $n$ is the index of the considered nearest neighbor. For the $2-4 \mathrm{~L}$ systems, $J_{n}$ 's vary depending on the pair position in the sample. We list them top to bottom as from outermost to innermost layer of sample. The last column gives the obtained critical temperature, $T_{c}$.

\begin{tabular}{lcccccc}
\hline \hline & $\begin{array}{c}J_{1} \\
(\mathrm{meV})\end{array}$ & $\begin{array}{c}J_{2} \\
(\mathrm{meV})\end{array}$ & $\begin{array}{c}J_{3} \\
(\mathrm{meV})\end{array}$ & $\begin{array}{c}J_{4} \\
(\mathrm{meV})\end{array}$ & $\begin{array}{c}J_{5} \\
(\mathrm{meV})\end{array}$ & $\begin{array}{c}T_{c} \\
(\mathrm{~K})\end{array}$ \\
\hline Bulk & 1.20 & 1.31 & 10.37 & 7.06 & 0.50 & 281 \\
\hline 4L & 0.58 & 1.64 & 16.72 & 10.26 & -0.21 & 285 \\
& 1.05 & 1.09 & 10.13 & 7.83 & 0.64 & \\
& 1.02 & 1.45 & 8.56 & 6.91 & 0.56 & \\
& & & & 7.01 & 0.51 & \\
& & & & 6.89 & & \\
\hline $3 \mathrm{~L}$ & 0.47 & 1.63 & 16.51 & 10.15 & -0.33 & 297 \\
& 0.92 & 1.09 & 8.29 & 10.03 & 0.52 & \\
& & 1.35 & & 6.89 & 0.45 & \\
& & & & 6.63 & & \\
\hline $2 \mathrm{~L}$ & 0.37 & 1.73 & 16.44 & 9.79 & -0.67 & 299 \\
& & 0.83 & 8.05 & 7.32 & 0.43 & \\
\hline $1 \mathrm{~L}$ & 0.94 & 2.13 & 11.88 & & & 326 \\
\hline \hline
\end{tabular}

ferromagnetic transition as compared to reality. To accommodate this issue, we rescaled all exchange parameters by a constant factor (0.65) so that the theoretically obtained $T_{c}=281 \mathrm{~K}$ is close to the experimentally measured $T_{c}$ of $265 \mathrm{~K}$ for bulk hematite. $28 \mid 29$

For each structure, starting from $2500 \mathrm{~K}$, the system is cooled down to $0 \mathrm{~K}$, and the average absolute magnetic moment per magnetic site, $\langle|M|\rangle$, is obtained as a function of temperature, as shown in Fig. 6(b). From the point where the magnetization curve exhibits maximal curvature as a function of temperature, we determine the critical temperature $\left(T_{c}\right)$ at which the system goes from weak ferromagnetic to the anti-ferromagnetic state, as listed in Table II. Of course, the behavior as a function of temperature is also very instructive, as will be discussed further on.

In general, as shown in Fig. 6(b), the magnetization curves exhibit similar behavior for all samples, with overall magnetization decrease with sample thickness. All curves exhibit a strong curvature in the range $700-860 \mathrm{~K}$ where the anti-ferromagnetic exchange interaction starts to take over. For different samples, the anti-ferromagnetic states gain dominance at temperatures 270-350 K. It should be pointed out that our MC simulation cannot capture the Néel temperature $\left(T_{N}\right)$ of $961 \mathrm{~K}^{28}$ for bulk hematite, due to the Ising approximation in which all spin magnetic moments are considered collinear (causing also the high-temperature ferromagnetism as discussed previously).

We start the discussion from the bulk sample, where the magnetization (orange dots in Fig. 6(b)) gradually decreases from on-site average of $\sim 0.12 \mu_{\mathrm{B}}$ at $1500 \mathrm{~K}$ down to $0.10 \mu_{\mathrm{B}}$ at $854 \mathrm{~K}$, where the anti-ferromagnetic state starts to build up. $T_{c}$ is found to be $281 \mathrm{~K}$ and the magnetization at room temperature is found to be $0.004 \mu_{\mathrm{B}}$, which is consistent with the experimentally 
determined value of $0.005 \mu_{\mathrm{B}} \cdot 29$ The magnetization of $4 \mathrm{~L}$ structure (blue dots in Fig. 6(b)) is slightly above the one of bulk. The antiferromagnetic interactions become apparent at $\sim 816 \mathrm{~K}$ and dominate below $T_{c}=285$ $\mathrm{K}$, which is nearly the same as for bulk. The magnetization for 3L sample exhibits the same behavior as $4 \mathrm{~L}$ and bulk structures, with slightly higher magnetization. The influence of the anti-ferromagnetic exchange arises at $\sim 771 \mathrm{~K} . T_{c}$ is found to be $297 \mathrm{~K}$, which can be considered as a significant jump as compared to bulk and $4 \mathrm{~L}$ samples. The results for $2 \mathrm{~L}$ system are also similar to thicker samples in terms of the form of the magnetization curve, with further increased magnetization compared to thicker samples. The anti-ferromagnetic exchange interaction is taking over at $790 \mathrm{~K}$, and $T_{c}$ is found to be 299 K.

The most significant changes are obtained when the sample is thinned down to a monolayer, even though the behavior of the magnetization curve still resembles the one found for thicker samples. The weak ferromagnetism is largest of all samples and the anti-ferromagnetic interaction rises at $\sim 711 \mathrm{~K}$ which is a significantly lower threshold temperature compared to $2 \mathrm{~L}$ and $3 \mathrm{~L}$ samples. The critical temperature of $T_{c}=326 \mathrm{~K}$ is above the room temperature and the highest of all considered sample thicknesses.

Our MC simulation therefore reveals that considering $T_{c}$ values, bulk and $4 \mathrm{~L}$ system on one side, and $2 \mathrm{~L}$ and $3 \mathrm{~L}$ systems on the other, are similar and can be considered equivalent. Monolayer hematene is different from thicker structures in all aspects, which is expected due to structural differences (cf. Fig. 1). Here it should be noted that our results do not support the findings of Ref 21, where FM to AFM transition was not observed for few-layer 2D $\alpha-\mathrm{Fe}_{2} \mathrm{O}_{3}$ down to low temperature. In addition to that, we speculate that in the experiment AFM phase could be further suppressed by the substrate effects so the system can preserve ferromagnetic behavior at even lower temperature. We reiterate the fact that true monolayer hematene exhibits much higher $T_{c}$ than 2-3L structures, so Morin transition should be experimentally observable there with lowered temperatures.

To complete the discussion, the magnetic anisotropy energies (MAE) of monolayer hematene were investigated as well. In Fig. 6(c), the MAE is projected on a sphere surface with respect to magnetization direction. There, $x, y$, and $z$ directions correspond to arm-chair, zig-zag, and out-of-plane directions, respectively. The minimum energy direction is found to be parallel to the $z$, i.e. to the out-of-plane direction, and the corresponding energy is set to $0.0 \mathrm{meV}$. MAE is found to be $0.34 \mathrm{meV}$ when the magnetization is parallel to the plane of the structure and does not change with the horizontal angle.

\section{CONCLUSIONS}

In summary, motivated by the recent experimental synthesis of few-layer thick hematite, i.e. $2 \mathrm{D} \alpha-\mathrm{Fe}_{2} \mathrm{O}_{3}, \frac{21}{2}$ we investigated theoretically the structural, vibrational, magnetic, and electronic properties of monolayer, fewlayer and bulk $\alpha-\mathrm{Fe}_{2} \mathrm{O}_{3}$. The monolayer $\alpha-\mathrm{Fe}_{2} \mathrm{O}_{3}$, for which we reserved the name hematene, was shown to indeed be stable, although not yet seen experimentally. Our calculated Raman spectrum shows that hematene can be clearly distinguished from few-layer and thicker structures, since the frequency shifts (weak when thinning the sample) are found to be the largest between monolayer and two-layer samples. We also find that all $2 \mathrm{D}$ structures exhibit distinctive Raman active modes in high frequencies that are absent in bulk.

In the electronic band structure, hematene exhibits strong spin asymmetry. Valence-band maximum (VBM), mid-gap bands and conduction-band minimum all belong to one spin state. VBM is dominated by $p_{x}$ and $p_{y}$ orbitals of oxygen, while the mid-gap band originates mostly from the $d_{z^{2}}$ orbital of surface iron atoms.

Our calculated optical spectra of monolayer, few-layer, and bulk structures show that the prominent part of the optical spectrum shifts to higher energy when the system is thinned down, as seen in experiment. 2-4 layer structures also exhibit a small step at the onset energy of the prominent peak, also seen in experiment, originating from the transition between the $p_{x}$ and $p_{y}$ of oxygen atoms to $d_{z^{2}}$ of surface iron. The similar type of transition is responsible for the mid-gap peak of a monolayer structure, whose separation from the prominent peak is another feature to distinguish true hematene from fewlayer samples.

Finally, our magnetic simulations based on DFTdetermined magnetic exchange parameters and subsequent Monte-Carlo simulations show that all considered structures display transitions from weak ferromagnetic to anti-ferromagnetic state with decreasing temperature. We note however that monolayer hematene exhibits higher $T_{c}$ in our calculations, hence there the transition to AFM state should be easier to detect experimentally.

Owing to its tunability with atomistic changes in thickness, magnetism in a broad temperature range (including room temperature), optical bandgap in the visible range, as well as the spin-dependent electronic and optical properties, 2D $\alpha-\mathrm{Fe}_{2} \mathrm{O}_{3}$ puts itself forward as a candidate for diverse technological applications. Taking all our findings at face value, we conclude that $2 \mathrm{D} \alpha-\mathrm{Fe}_{2} \mathrm{O}_{3}$ is certainly worth of further investigation and use in functional heterostructures.

\section{Acknowledgments}

This work was supported by Research FoundationFlanders (FWO-Vlaanderen). Computational resources 
were provided by Flemish Supercomputer Center (VSC), and TUBITAK ULAKBIM, High Performance and Grid Computing Center (TR-Grid e-Infrastructure). Part of this work was also supported by FLAG-ERA project
TRANS-2D-TMD and TOPBOF-UAntwerp. M. Y. was supported by a postdoctoral fellowship from the Flemish Science Foundation (FWO-Vl).
* Electronic address: milorad.milosevic@uantwerpen.be

1 K. S. Novoselov, A. K. Geim, S. V. Morozov, D. Jiang, Y. Zhang, S. V. Dubonos, I. V. Grigorieva, and A. A. Firsov, Science 306, 666 (2004).

2 K. S. Novoselov, A. K. Geim, S. V. Morozov, D. Jiang, M. I. Katsnelson, I. V. Grigorieva, S. V. Dubonos, and A. A. Firsov, Nature 438, 197 (2005).

${ }^{3}$ H. Sahin, S. Cahangirov, M. Topsakal, E. Bekaroglu, E. Akturk, R. T. Senger, and S. Ciraci, Phys. Rev. B 80, 155453 (2009).

${ }^{4}$ Q. Wang, Q. Sun, P. Jena, and Y. Kawazoe, ACS Nano 3, 621 (2009).

${ }^{5}$ K. K. Kim, A. Hsu, X. Jia, S. M. Kim, Y. Shi, M. Hofmann, D. Nezich, J. F. Rodriguez-Nieva, M. Dresselhaus, T. Palacios, and J. Kong, Nano Lett. 12 (1), 161 (2012).

${ }^{6}$ P. Tsipas, S. Kassavetis, D. Tsoutsou, E. Xenogiannopoulou, E. Golias, S. A. Giamini, C. Grazianetti, D. Chiappe, A. Molle, M. Fanciulli, and A. Dimoulas, Appl. Phys. Lett. 103, 251605 (2013).

7 C. Bacaksiz, H. Sahin, H. D. Ozaydin, S. Horzum, R. T. Senger, and F. M. Peeters, Phys. Rev. B 91, 085430 (2015).

8 R. A. Gordon, D. Yang, E. D. Crozier, D. T. Jiang, and R. F. Frindt, Phys. Rev. B 65, 125407 (2002).

9 J. N. Coleman, M. Lotya, A. O’Neill, S. D. Bergin, P. J. King, U. Khan, K. Young, A. Gaucher, S. De, R. J. Smith, I. V. Shvets, S. K. Arora, G. Stanton, H. Y. Kim, K. Lee, G. T. Kim, G. S. Duesberg, T. Hallam, J. J. Boland, J. J. Wang, J. F. Donegan, J. C. Grunlan, G. Moriarty, A. Shmeliov, R. J. Nicholls, J. M. Perkins, E. M. Grieveson, K. Theuwissen, D. W. McComb, P. D. Nellist, and V. Nicolosi, Science 331, 568 (2011).

10 Q. H. Wang, K. Kalantar-Zadeh, A. Kis, J. N. Coleman, and M. S. Strano, Nat. Nanotech. 7, 699 (2012).

11 J. S. Ross, P. Klement, A. M. Jones, N. J. Ghimire, J. Yan, D. G. Mandrus, T. Taniguchi, K. Watanabe, K. Kitamura, W. Yao, D. H. Cobden, and X. Xu, Nat. Nanotech. 9, 268 (2014).

12 H. Sahin, S. Tongay, S. Horzum, W. Fan, J. Zhou, J. Li, J. Wu, and F. M. Peeters, Phys. Rev. B 87, 165409 (2013).

13 S. Tongay, H. Sahin, C. Ko, A. Luce, W. Fan, K. Liu, J. Zhou, Y. S. Huang, C. H. Ho, J. Yan, D. F. Ogletree, S. Aloni, J. Ji, S. Li, J. Li, F. M. Peeters, and J. Wu, Nat. Comm. 5, 3252 (2014).

14 S. Horzum, D. Cakir, J. Suh, S. Tongay, Y. S. Huang, C. H. Ho, J. Wu, H. Sahin, and F. M. Peeters, Phys. Rev. B 89, 155433 (2014).

15 B. Chen, H. Sahin, A. Suslu, L. Ding, M. I. Bertoni, F. M. Peeters, and S. Tongay, ACS Nano 9 (5), 5326 (2015).

16 S. Cahangirov, M. Topsakal, E. Akturk, H. Sahin, and S. Ciraci, Phys. Rev. Lett. 102, 236804 (2009).

17 A. Kara, H. Enriquez, A. P. Seitsonen, L. C. L. Y. Voon, S. Vizzini, B. Aufray, and H. Oughaddou, Surf. Science Report. 67, 1 (2012).

18 V. Kochat, A Samanta, Y. Zhang, S. Bhowmick, P. Manimunda, S. Asif, A. S. Stender, R. Vajtai, A. K. Singh, C.
S. Tiwary, and P. M. Ajayan, Sci. Adv. 4, e1701373 (2018).

19 S. V. Badalov, M. Yagmurcukardes, F. M. Peeters, and H. Sahin, J. Phys. Chem. C 122, 28302 (2018).

20 M. Nakhaee, M. Yagmurcukardes, S. A. Ketabi, and F. M. Peeters, Phys. Chem. Chem. Phys. 21, 15798 (2019).

21 A. P. Balan, S. Radhakrishnan, C. F. Woellner, S. K. Sinha, L. Deng, C. de los Reyes, B. M. Rao, M. Paulose, R. Neupane, A. Apte, V. Kochat, R. Vajtai, A. R. Harutyunyan, C.-Wu Chu, G. Costin, D. S. Galvao, A. A. Marti, P. A. van Aken, O. K. Varghese, C. S. Tiwary, A. M. M. R. Iyer, and P. M. Ajayan, Nat. Nanotech. 13, 602 (2018).

22 E. Kan, M. Li, S. Hu, C. Xiao, H. Xiang, and K. Deng, J. Phys. Chem. Lett. 4, 1120 (2013).

23 M. Marelli, A. Naldoni, A. Minguzzi, M. Allieta, T. Virgili, G. Scavia, S. Recchia, R. Psaro, and V. Dal Santo, ACS Appl. Mater. Interfaces 6, 11997 (2014).

24 M. Mishra and D.-M. Chun, Appl. Catal. A 6, 126 (2015).

25 J. Chen, L. Xu, W. Li, and X. Gou, Adv. Mater. 17, 582 (2005).

26 H. Zeng, J. Li, J. P. Liu, and Z. L. Wang, Nature 420, 395 (2002).

27 J. Katz, S. C. Riha, N. C. Jeong, A. B. F. Martinson, K. Omar, O. K. Farha, and J. T. Hupp, Coord. Chem. Rev. 256, 2521 (2012).

${ }^{28}$ F. J. Morin, Phys. Rev. 78, 819 (1950).

29 T. Moriya, Phys. Rev. 120, 91 (1960).

30 R. D. L. Smith, M. S. Prevot, R. D. Fagan, Z. Zhang, P. A. Sedach, M. K. J. Siu, S. Trudel, and C. P. Berlinguette, Science 340, 60 (2013).

31 S. Sakurai, J.-I. Shimoyama, K. Hashimoto, and S. I. Ohkoshi, Chem. Phys. Lett. 458, 333 (2008).

32 A. Namai, M. Yoshikiyo, K. Yamada, S. Sakurai, T. Goto, T. Yoshida, T. Miyazaki, M. Nakajima, T. Suemoto, H. Tokoro, and S. I. Ohkoshi, Nat. Commun. 3, 1035 (2012).

33 S. I. Ohkoshi, S. Kuroki, S. Sakurai, K. Matsumoto, K. Sato, and S. Sasaki, Angew. Chem., Int. Ed. 46, 8392 (2007).

34 A. Namai, S. Sakurai, M. Nakajima, T. Suemoto, K. Matsumoto, M. Goto, S. Sasaki, and S. I. J. Ohkoshi, Am. Chem. Soc. 131, 1170 (2009).

35 N. V. Long, Y. Yang, M. Yuasa, C. M. Thi, Y. Q. Cao, T. Nann, and M. Nogami, RSC Advances 15, 6383 (2014).

${ }^{36}$ X. L. Wu, Y. G. Guo, L. J. Wan, and C. W. Hu, J. Phys. Chem. C 112, 16824 (2008).

37 A. Banerjee, V. Aravindan, S. Bhatnagar, D. Mhamane, S. Madhavi, and S. Ogale, Nano Energy 2 (5), 890 (2013).

38 X. Gu, L. Chen, Z. C. Ju, H. Y. Xu, J. Yang, and Y. T. Qian, Adv. Funct. Mater. 23, 4049 (2013).

39 W. S. Tang, Y. Su, Q. Li, S. A. Gao, and J. K. Shang, Water Res. 47, 3624 (2013).

40 C. Y. Cao, J. Qu, W. S. Yan, J. F. Zhu, Z. Y. Wu, and W. G. Song, Langmuir 28, 4573 (2012).

41 J. X. Wang, R. S. Yuan, L. Y. Xie, Q. F. Tian, S. Y. Zhu, Y. H. Hu, P. Liu, X. C. Shi, and D. H. Wang, RSC Advances 2, 1112 (2012). 
42 G. H. Qiu, H. Huang, H. Genuino, N. Opembe, L. Stafford, S. Dharmarathna, and S. L. Suib, J. Phys. Chem. C 115, 19626 (2011).

43 J. L. Dormann, D. Fiorani, and E. Tronc, Adv. Chem. Phys. 98, 283 (1997).

44 R. D. Zysler, D. Fiorani, A. M. Testa, M. Godinho, E. Agostinelli, and L. Suber, J. Magn. Magn. Mater. 272, 1575 (2004).

45 D. Schroeer and R. C. Nininger, Phys. Rev. Lett. 19, 632 (1967).

46 M. Sorescu, R. A. Brand, D. Mihaila-Tarabasanu, and L. Diamandescu, J. Appl. Phys. 85, 5546 (1999).

47 R. I. Gonzalez, J. Mella, P. Díaz, S. Allende, E. E. Vogel, C. Cardenas, and F. Munoz, 2D Mater. 6045002 (2019).

48 A. C. M. Padilha, M. Soares, E. R. Leite, and A. Fazzio, J. Phys. Chem. C 123, 16359 (2019).

49 B. Huang, G. Clark, E. Navarro-Moratalla, D. R. Klein, R. Cheng, K. L. Seyler, D. Zhong, E. Schmidgall, M. A. McGuire, D. H. Cobden, W. Yao, D. Xiao, P. JarilloHerrero, and X. Xu, Nature 546, 270 (2017).

${ }^{50}$ G. Kresse and J. Hafner, Phys. Rev. B 47, 558 (1993).

51 G. Kresse and J. Furthmuller, Phys. Rev. B 54, 11169 (1996).

52 J. P. Perdew, K. Burke, and M. Ernzerhof, Phys. Rev. Lett. 77, 3865 (1996).

53 S. J. Grimme, J. Comput. Chem. 27, 1787 (2006).

54 S. L. Dudarev, G. A. Botton, S. Y. Savrasov, C. J. Humphreys and A. P. Sutton, Phys. Rev. B 57, 1505
(1998)

55 G. Henkelmana, A. Arnaldssonb, H. Jónsson, Comput. Mater. Sci. 36, 354 (2006).

56 D. Alfe, Comput. Phys. Commun. 180, 2622 (2009).

57 A. Fonari and S. Stauffer, "https://github.com/ramansc/VASP/" (2013).

58 M. Yagmurcukardes, C. Bacaksiz, E. Unsal, B. Akbali, R. T. Senger, and H. Sahin, Phys. Rev. B 97, 115427 (2018).

59 M. Yagmurcukardes, F. M. Peeters, and H. Sahin, Phys. Rev. B 98, 085431 (2018).

60 H. J. Xiang, E. J. Kan, S.-H. Wei, M.-H. Whangbo, and X. G. Gong, Phys. Rev. B 84, 224429 (2011).

61 D. Hayes, R. G. Hadt, J. D. Emery, A. A. Cordones, A. B. F. Martinson, M. L. Shelby, K. A. Fransted, P. D. Dahlberg, J. Hong, X. Zhang, Q. Kong, R. W. Schoenleinc, and L. X. Chen, Ener. Env. Sci. 9, 3754 (2016).

62 It should be noted that since Balan et al. ${ }^{21}$ synthesized samples by exfoliation in crystalline directions of [001] and [010], there is a possibility that their Raman spectrum has also contribution from few-layers of [010] phase, but we do not expect significant differences in Raman activity of few-layer [001] and [010] hematite.

63 It should also be noted that in calculation of electronic properties of a real semiconductor, independently from the smearing parameter, the bands are fully occupied and Fermi level is always in the band gap. 